\title{
AVALIAÇÃO ESTEREOTÔMICA DE TEORES VIA MÉTODO DE MONTE CARLO
}

\author{
P. F. T. LOPES' ${ }^{1}$ e J. A. M. LUZ $^{2}$ \\ 'Departamento de Engenharia de Minas - Universidade Federal de Ouro Preto \\ pftlopes@yahoo.com.br ${ }^{1}$ - jaurelioluz@gmail.com ${ }^{2}$
}

Artigo submetido em novembro/2013 e aceito em março/2014

DOI: http://dx.doi.org/10.15628/holos.2014.1751

\section{RESUMO}

A utilização de gabaritos visuais de teores é uma ferramenta por vezes utilizada, principalmente em atividades de campo, para a avaliação prévia e estimativa de teores e concentração de fases visualmente distintas. Estes padrões geométricos de superfície podem ser simulados com métodos estatísticos, possibilitando a utilização eficaz de outras ferramentas, como a análise computacional de imagens. Neste trabalho propõe-se a utilização do método de Monte Carlo para a simulação destes padrões (gabaritos), usando-se previamente a modelagem matemática das fases mineralógicas/tipológicas imersas no maciço. Apenas alguns parâmetros das distribuições estatísticas associadas à distribuição espacial das fases constituintes são alterados, permitindo uma correlação entre valores desses parâmetros e o padrão dos gabaritos. Tal ferramenta serve, por exemplo, para estimação expedita de teor de blocos de lavra, a partir da análise visual das faces expostas da bancada, bem como para análise petrográfica ou metalográfica

PALAVRAS-CHAVE: gabarito visual, estereotomia, método estocástico, números pseudoaleatórios.

\section{STEREOTOMIC EVALUATION OF CONTENT VIA MONTE CARLO METHOD}

\begin{abstract}
The use of visual templates for content calculation is a useful tool, especially in field activities, prior to the evaluation and estimation the amount of useful materials and visually distinct phases. These geometric surface patterns can be simulated by statistical methods, enabling the effective use of other tools such as computational imaging. In this paper we propose the use of Monte Carlo method for simulation of these patterns (templates), using previously mathematical
\end{abstract}

modeling of mineralogical/typological phases disseminated in a matrix. Only some parameters of statistical distributions linked to spatial distribution of the constituent phases are changed, allowing correlation between values of these parameters and the standards of surface pattern. Such a tool serves, for instance, to expeditiously estimation of ore contents in exploitable blocks, from visual analysis of the exposed bench faces, or to petrographic or metallographic analyses as well.

KEYWORDS: visual template, stereology, stochastic method, pseudorandom numbers. 


\section{INTRODUÇÃO}

Em algumas instâncias da lavra, em especial a céu aberto, o engenheiro de minas deparase com frentes onde o mineral minério acha-se preenchendo planos de fraturas preexistentes, ou onde o mineral de interesse aparece disperso ao longo da superfície da face da escavação. Em serpentinitos mineralizados, a crisotila é um exemplo clássico de tal ocorrência. Essas fraturas podem ser modeladas como placas idealizadas com atitude e espessura variadas, segundo distribuições probabilidade determinadas pelas condições genéticas do jazimento, tais como tectonismo e esquema de percolação de fluidos mineralizantes. Outro exemplo do ponto de vista prático é a utilização de gabaritos de estimação de teor visual, para estimativa da composição de rochas, em campo.

O objetivo deste trabalho é desenvolvimento de um modelo utilizando-se de técnicas de modelagem geométrica e utilização de distribuições estatísticas para simular as principais características de materiais heterogêneos, em especial os metalográficos e geológicos. Com os resultados destas simulações, são mostrados cenários visuais para facilitar tomadas de decisões a curto prazo sobre o sequenciamento de lavra.

No modelo construído foram tomadas as seguintes considerações:

- O modelo tem como objetivo a geração de cenários plausíveis, as leis de distribuições de probabilidade previamente assumidas, o usuário terá que fornecer os dados tratados com antecedência por algum sistema computacional estatístico.

- O modelo tridimensional gerado é composto por elementos espaciais discretos e idealizados, e utiliza parâmetros fornecidos pelo usuário.

- O processo de modelagem é uma arte, e depende de fatores subjetivos. Implicando que o modelo apresentado não é único e não pretende esgotar o assunto, corresponde apenas a uma das possíveis abstrações do problema abordado. Para exemplificar esse aspecto, descreve-se aqui, adicionalmente, uma abordagem simplificada (implementada em Pascal, utilizando a plataforma de desenvolvimento Lazarus).

\section{O MÉTODO DE MONTE CARLO}

Métodos de Monte Carlo (ou experimentos de Monte Carlo) são uma classe de algoritmos computacionais que dependem de amostragem aleatória repetida para calcular os seus resultados. Métodos de Monte Carlo são frequentemente utilizados em simulações de computador de sistemas físicos e matemáticos. Estes métodos são mais adequados para o cálculo de um computador, e tendem a ser utilizado quando é impossível calcular resultado exato com um algoritmo determinista. Este método é também utilizado para complementar derivações teóricas (Metropolis e colaboradors, 1953; Hammsersley e Handscomb, 1967; Hillier e Lieberman, 2005; Sobol, 1983, Hromkovic, 2001). 
Métodos de Monte Carlo são especialmente úteis para a simulação de sistemas com muitos graus de liberdade acoplados, como os fluidos, materiais sólidos desordenados, e estruturas celulares. Eles são usados para modelar fenômenos com incerteza significativa em insumos, tais como o cálculo do risco de empreendimentos econômicos (Fernandes, 2005). Eles são amplamente utilizados em matemática, por exemplo, para avaliar integrais definidas multidimensionais, com condições de contorno complicadas. Quando simulações de Monte Carlo foram aplicadas na exploração do espaço e exploração de petróleo, suas previsões de falhas, excessos de custos e atrasos no cronograma são rotineiramente melhor do que a intuição humana ou métodos alternativos simplificados.

O nome "Monte Carlo" surgiu durante o projeto Manhattan na Segunda Guerra Mundial. No projeto de construção da bomba atómica, Ulam, von Neumann e Fermi consideraram a possibilidade de utilizar o método, que envolvia a simulação direta de problemas de natureza probabilística relacionados com o coeficiente de difusão do nêutron em certos materiais. Apesar de ter despertado a atenção desses cientistas em 1948, a lógica do método já era conhecida há bastante tempo. Por exemplo, existe um registro de um artigo escrito por Lorde Kelvin dezenas de anos antes, que já utilizava técnicas de Monte Carlo em uma discussão das equações de Boltzmann. Um problema típico que usa técnicas de Monte Carlo ainda mais antigo é o da chamada agulha de Buffon (Sobol, 1983)

\section{MODELO MATEMÁTICO MATRICIAL}

Para simular uma distribuição espacial de uma determinada espécie ou componente mineral, são gerados 3 vetores com $n$ elementos cada, simulando o distribuição unidimensional desta espécie, a partir de uma dada distribuição de probabilidade. Em princípio, esta distribuição pode ser de qualquer natureza, desde que resulte em valores entre 0 e 1 , ou que tenha seus valores expressos em termos de coordenadas reduzidas, mas mantendo os limites binários.

Desta forma, constroem-se os vetores:

$$
\vec{a}=\left(\begin{array}{c}
a_{1} \\
a_{2} \\
\vdots \\
a_{n}
\end{array}\right) \quad \vec{b}=\left(\begin{array}{c}
b_{1} \\
b_{2} \\
\vdots \\
b_{n}
\end{array}\right) \quad \vec{c}=\left(\begin{array}{c}
c_{1} \\
c_{2} \\
\vdots \\
c_{n}
\end{array}\right)
$$

Onde:

$$
a_{i}, b_{i}, c_{i} \in f d p(i) \rightarrow[0,1]
$$

Construídos os vetores $\vec{a}, \vec{b}$ e $\vec{c}$, é gerada uma matriz tridimensional, tal como representado na Figura 1, que representa a distribuição espacial dos $n^{3}$ elementos discretos que compõe a estrutura geológica simulada. Assim temos:

$$
D=\left(\vec{a} \cdot \vec{b}^{T}\right) \cdot \vec{c}^{T}
$$


Onde:

$D \leftarrow d_{i, j}^{k} \mid d_{i, j}^{k}=a_{i} \cdot b_{j} \cdot c_{k}$

A transposição dos vetores simulados é feita de modo a compatibilizar o sistema de matrizes, criando um espaço tridimensional discreto de tamanho $n \times n \times n$. Nota-se que esta formulação serve tanto para domínios ou materiais isotrópicos quanto para anisotrópicos. No caso isotrópico, basta se utilizar a mesma função de distribuição de probabilidade $(f d p)$ nas 3 direções do espaço tridimensional. Já no caso anisotrópico, basta utilizar-se uma $f d p$ adequada para cada direção, caracterizando, portanto, a anisotropia constitucional do sistema.

$\begin{array}{cccccc} & & d_{1,1}^{n} & d_{2,1}^{n} & \cdots & d_{n, 1}^{n} \\ & \therefore & \therefore & \therefore & \ddots & d_{n, 2}^{n} \\ d_{1,1}^{1} & d_{2,1}^{1} & \cdots & d_{n, 1}^{1} & \therefore & \vdots \\ d_{1,1}^{1} & d_{2,2}^{2} & \cdots & d_{n, 2}^{1} & \ddots & d_{n, n}^{n} \\ \vdots & \vdots & \ddots & \vdots & \therefore & \\ d_{1, n}^{1} & d_{2, n}^{1} & \cdots & d_{n, n}^{1} & & \end{array}$

Figura 1 - Matriz tridimensional simulada.

Por fim, para estimar-se o teor global da espécie em questão, a qual sua probabilidade de ocorrência foi simulada a partir das $n^{3}$ variáveis espaciais aleatórias, basta se calcular a média aritmética dos valores da matriz tridimensional $D$. O resultado é o próprio teor da espécie, sem demais ajustes, dado que foi imposta os limites binários para $d_{i, j}^{k}$. Assim temos:

$$
\mu=\frac{1}{n^{3}} \cdot \sum_{i=0}^{n} d_{i, i}^{i}
$$

No caso da avaliação bidimensional de uma dada distribuição espacial da espécie mineralógica, basta reduzir o método em termos apenas dos vetores $\vec{a}$ e $\vec{b}$. A construção da matriz, agora bidimensional, será o produto dos dois vetores orientados segundo direções perpendiculares, e o teor final simulado, a média dos termos obtidos (Lima, 2006).

\section{SIMULAÇÃO E RESULTADOS}

Para ilustrar o método proposto, foi simulada uma espécie mineralógica hipotética, distribuída espacialmente segundo uma distribuição binomial, ou seja, a espécie possui, num determinado ponto do espaço, a chance $\lambda$ de ocorrer segundo uma direção. Foi utilizado um tamanho de vetor $n=200$, gerando um total de $n^{3}=8.000 .000$ de elementos discretos 
distribuídos espacialmente, para cada valor de $\lambda$. O intervalo de variação do parâmetro $\lambda$ (passo) foi de $10 \%$, resultando valores desde $0 \%$ até $100 \%$. Empregou-se o software comercial Mathcad 15.0 (da Parametric Technology Corporation, 2010), através dos seguintes algoritmos, generalizados:

$$
D_{2 D}=\operatorname{rbinom}(n, 1, \lambda) \cdot \operatorname{rbinom}(n, 1, \lambda)^{T}
$$

Para o caso bidimensional, e:

$$
D_{3 D}=\left\{\begin{array}{c}
\text { for } i \in[0, n-1] \\
D_{i} \leftarrow\left(\operatorname{rbinom}(n, 1, \lambda) \cdot \operatorname{rbinom}(n, 1, \lambda)^{T}\right)^{\langle i\rangle} \cdot \operatorname{rbinom}(n, 1, \lambda)^{T}
\end{array}\right.
$$

Para o caso tridimensional, optou-se pela distribuição binomial, pois além da fácil implementação computacional, ela permite a formulação da média teórica (teor global da espécie), para o caso de $n \rightarrow \infty$. Assim, para o caso 2D e 3D, tem-se, respectivamente:

$$
\mu_{2 D}=\lambda^{2} \text { e } \mu_{3 D}=\lambda^{3}
$$

A partir das simulações realizadas foi construída a Tabela 1, e plotado o gráfico do parâmetro $\lambda$ em função do teor global. Também foi realizada uma interpolação não linear, de modo a se obter uma curva de ajuste dos pontos obtidos com a simulação.

O ajuste foi condicionado de modo a obter-se curva $y=a x^{n}$, análoga ao modelo teórico para o teor global da espécie. Nota-se um coeficiente de correlação dos valores, $R^{2}$, muito próximo de 1 , o que indica que o ajuste matemático obtido foi satisfatório, e que o tamanho de vetor, $n=200$ utilizado na simulação já é suficientemente grande não só para aproximarmos a simulação do modelo teórico, mas também para evitar flutuações do processo de geração de números aleatórios, intrínseco ao Método de Monte Carlo, e que poderia ocasionar desvios do modelo, em escalas de simulação muito pequenas. 
Tabela 1 - Resultados obtidos a partir da simulação

\begin{tabular}{c|c|c|c|c|c|c}
\hline & \multicolumn{2}{|c|}{ Simulação } & \multicolumn{2}{c|}{ Teórico } & \multicolumn{2}{c}{ Resíduo absoluto } \\
\hline$\lambda$ & $\mu 2 D$ & $\mu 3 D$ & $\mu^{\prime} 2 \mathrm{D}$ & $\mu^{\prime} 3 \mathrm{D}$ & $\mathrm{r} 2 \mathrm{D}$ & $\mathrm{r} \mathrm{D}$ \\
\hline 0 & 0 & 0 & 0 & 0 & 0 & 0 \\
\hline 0.1 & 0.008125 & 0.000805 & 0.01 & 0.001 & -0.00188 & -0.00019 \\
\hline 0.2 & 0.041475 & 0.006973 & 0.04 & 0.008 & 0.001475 & -0.00103 \\
\hline 0.3 & 0.09635 & 0.030883 & 0.09 & 0.027 & 0.00635 & 0.003883 \\
\hline 0.4 & 0.1611 & 0.068454 & 0.16 & 0.064 & 0.0011 & 0.004454 \\
\hline 0.5 & 0.24835 & 0.12313 & 0.25 & 0.125 & -0.00165 & -0.00187 \\
\hline 0.6 & 0.386975 & 0.218387 & 0.36 & 0.216 & 0.026975 & 0.002387 \\
\hline 0.7 & 0.493325 & 0.316978 & 0.49 & 0.343 & 0.003325 & -0.02602 \\
\hline 0.8 & 0.625325 & 0.508241 & 0.64 & 0.512 & -0.01468 & -0.00376 \\
\hline 0.9 & 0.804125 & 0.711928 & 0.81 & 0.729 & -0.00588 & -0.01707 \\
\hline 1 & 1 & 1 & 1 & 1 & 0 & 0 \\
\hline
\end{tabular}

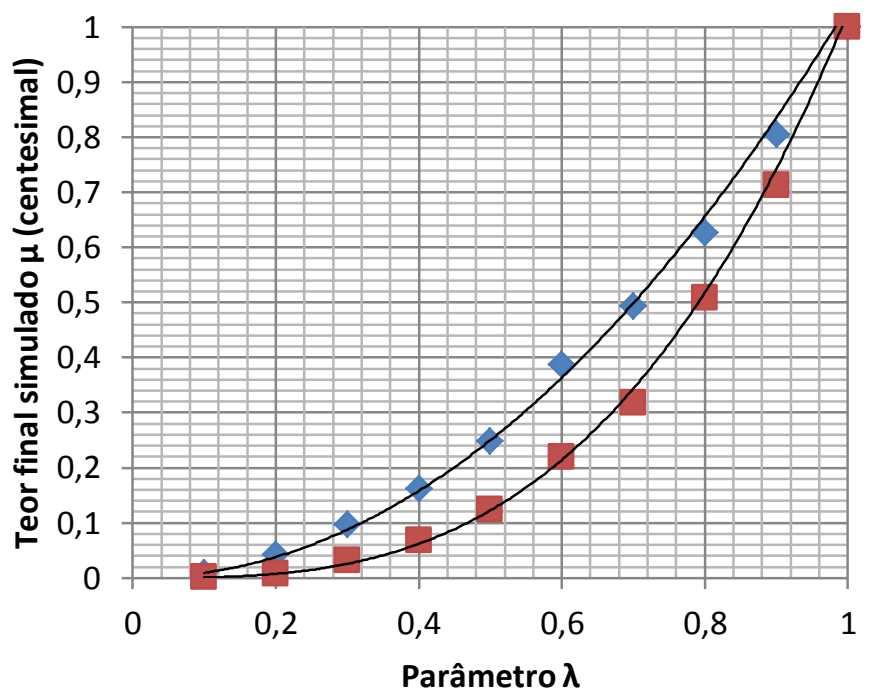

$\rightarrow 2 \mathrm{D}$

3D

- Potência (2D)

Potência (3D)

$y=1,0378 x^{2,0537}$

$\mathrm{R}^{2}=0,998$

$y=1,0265 x^{3,0667}$

$R^{2}=0,9985$

Figura 2 - Regressão não-linear dos resultados simulados.

A partir dos resultados simulados, foram analisados os resíduos absolutos do modelo simulado em relação ao modelo teórico, bem como plotado uma curva de aderência dos valores teóricos com os simulados, como pode ser observados nos dois gráficos da Figuras 3: 

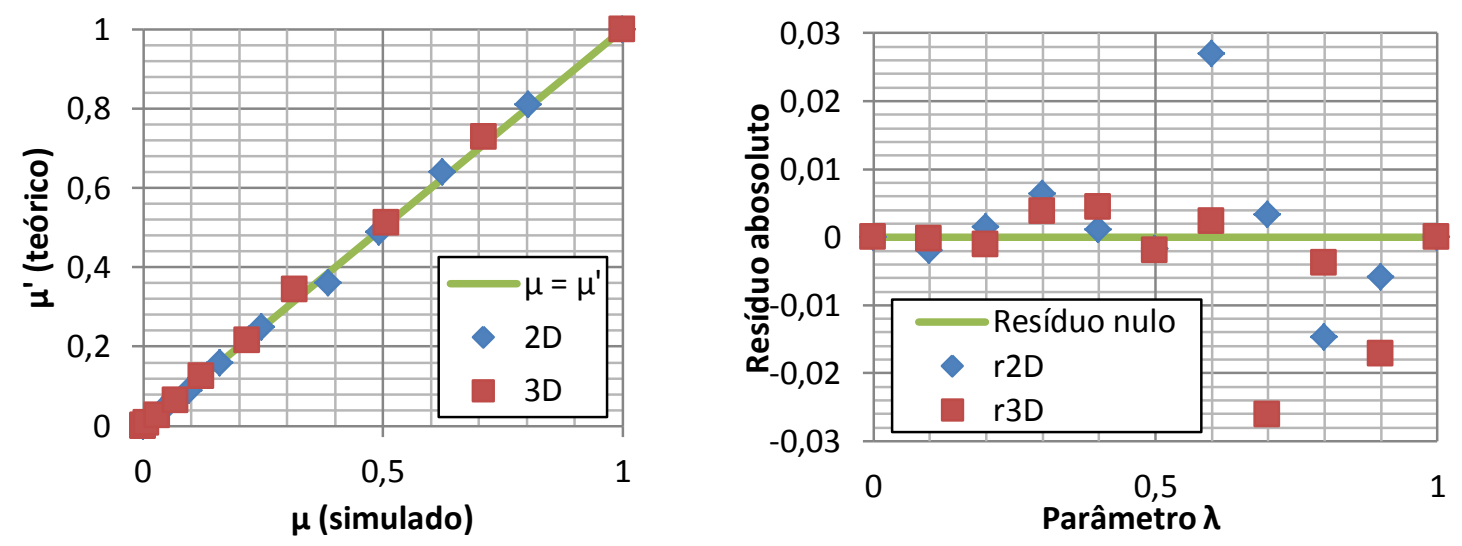

Figura 3 - Curva de aderência entre o modelo teórico e a simulação e análise dos resíduos absolutos obtidos pela simulação (ocorrendo dois resíduos aberrantes).

\section{ABORDAGEM VIA LAZARUS E PLANILHA ELETRÔNICA}

Abordagem de simulação em 2D de distribuição aleatória de partículas não aglomerantes foi feita, igualmente, empregando-se, por um lado, linguagem Free Object Pascal (da plataforma gratuita de ambiente de desenvolvimento integrado Lazarus, em tudo similar ao Delphi) e, por outro planilhas eletrônicas de cálculo (como o sistema gratuito Calc da suíte Libre Office, ou o Excel da Microsoft).

Como assinalam Spowart e colaboradores (2001), se o espaço amostral onde os centroides de partículas aleatoriamente dispostas e não sobreponentes for quadriculado (criando quadrículas com aresta igual a I), a densidade em cada quadrícula obedece à distribuição de Poisson, expressa por:

$p\left(n_{i}\right)=\frac{n_{i}^{\lambda} \times \exp (-\lambda)}{\lambda !}$

Onde:

$\lambda$ - densidade média para toda a área (número de pontos por quadrícula);

$\mathrm{n}_{\mathrm{i}}$ - número de pontos na quadrícula;

$p\left(n_{i}\right)$ - probabilidade de haver $n_{i}$ pontos na quadrícula em análise.

Ademais, para a distribuição de Poisson o desvio padrão populacional é igual à raiz quadrada do parâmetro $\lambda$ (média) da distribuição. Um algoritmo muito simples (podendo ter seu desempenho computacional muito melhorado) é mostrado na tabela 2, a seguir. Registre-se que a função ocorrencia $(p)$ do algoritmo foi implementada sorteando-se um número pseudoaleatório entre 0 e 1 (via comando random()) e comparando-o com a proporção $p$ : se random()>p então 0 valor é zero (não existe), caso contrário o valor é 1 (existe). 
Tabela 2 - Código em Pascal do módulo para geração de centroides aleatórios em retângulo.

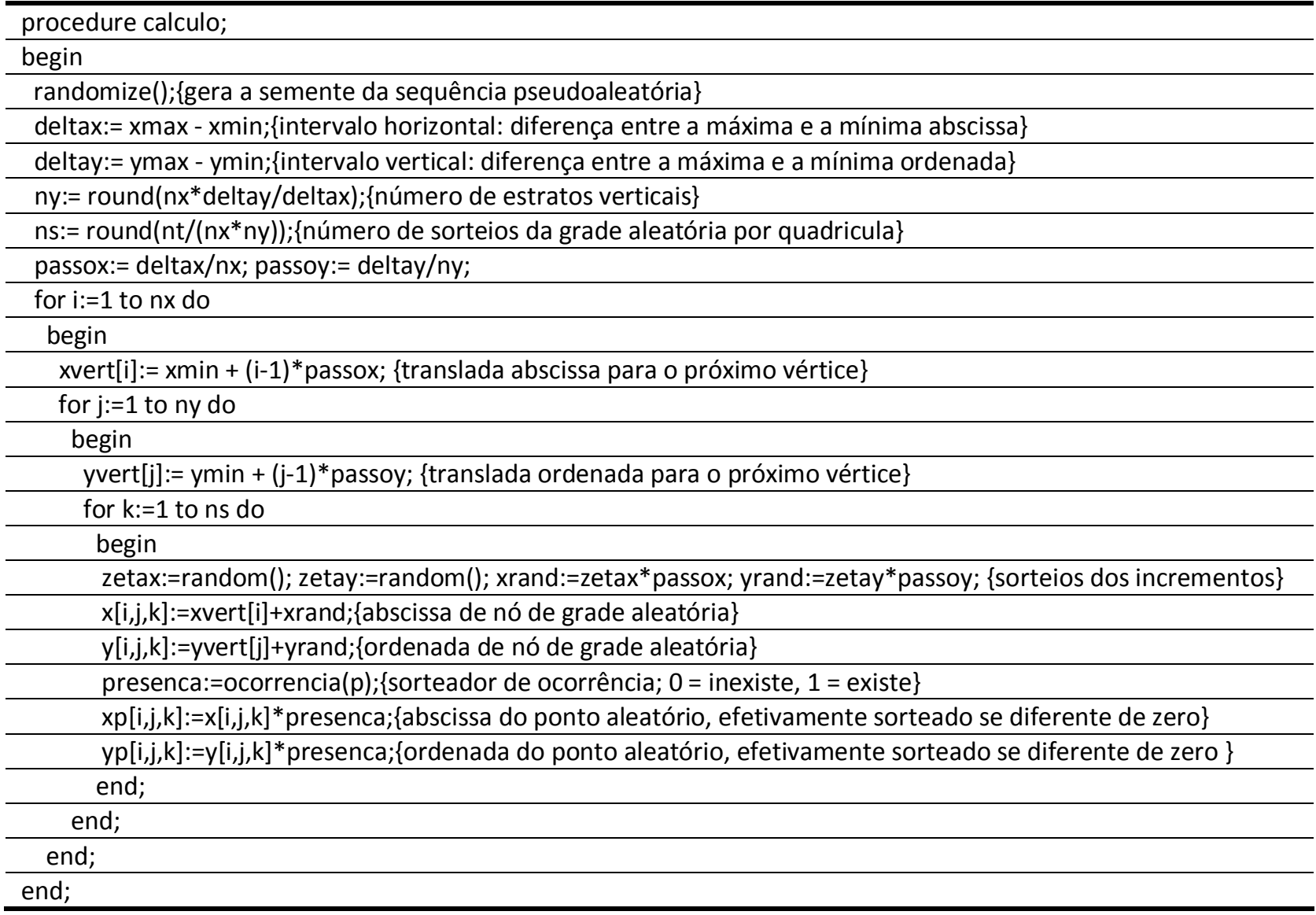

Para a implementação equivalente em planilha eletrônica deve-se trabalhar na mesma filosofia, baseando-se o método de Monte Carlo na função ALEATÓRIO (); nesse caso os contadores $i, j$ e $k$ podem ser substituídos por linhas (embora com muito menor flexibilidade computacional).

Visando a demonstrar graficamente os resultados obteníveis com a implementação do algoritmo da tabela anterior, plotaram-se duas seções simuladas, mostradas na Figura 4, juntamente com uma micrografia de um "compósito de alumínio reforçado descontinuamente (DRA)" estudado por Spowart (2006), para efeito comparativo. O diâmetro dos círculos simulados foi de $11,76 \%$ da aresta das quadrículas (linhas de grade principais, com espaçamento unitário). Na simulação da esquerda da figura sortearam-se 792 pontos em grade de tamanho [?x = 6 por ?]y $=10$ (grade de $6 \times 10$ quadrículas de área unitária), resultando proporção árica igual a 14,35 \%. Empregando a mesma grade e o mesmo tamanho dos círculos, a simulação central da figura 4 resultou da seleção de 1980 pontos sorteados aleatoriamente, mas estratificados em seis estratos horizontais (e 10 verticais), acarretando proporção árica de 35,87 \%.

Com vistas à calibração e/ou validação do sistema, as secções simuladas na figura 4 foram submetidas adicionalmente à análise de histograma (frequência de distribuição) de pixels no sistema gratuito de tratamento de imagem Irfanview, acusando os teores de 17,88 \% e 34,90\%. 


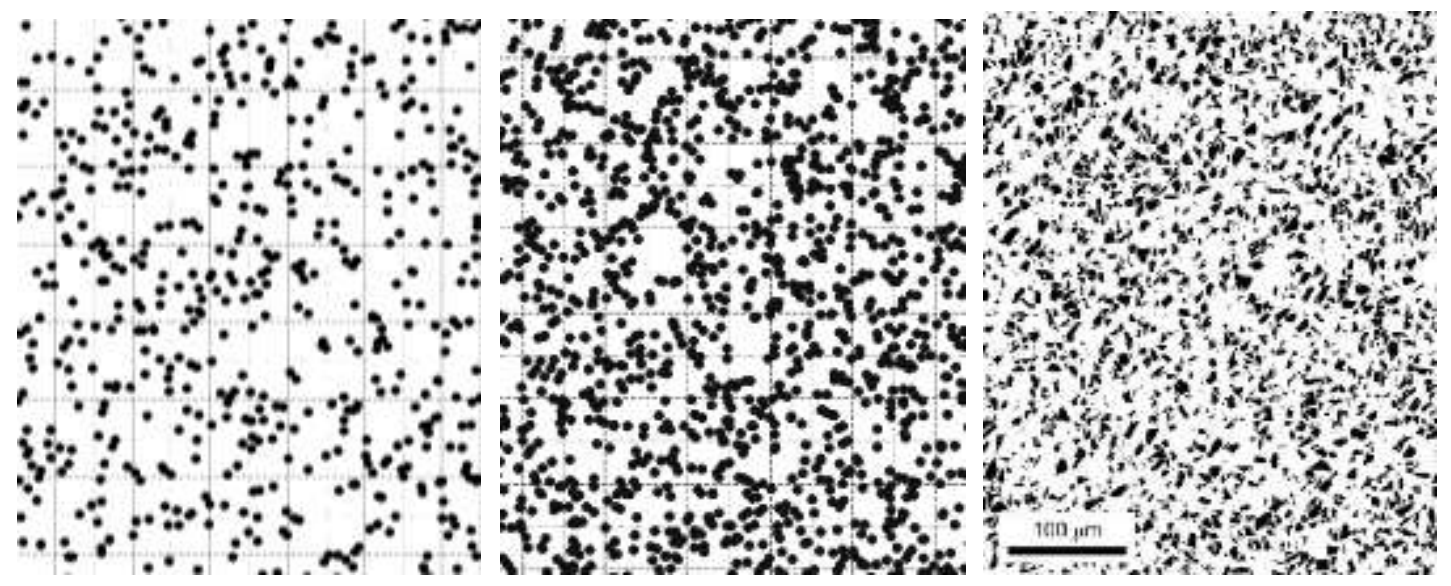

Figura 4 - Seções aleatórias, para proporções: $p=14,35 \%$ e $p=35,87 \%$ (coordenadas geradas em Lazarus); à direita, seção metalográfica (micrografia de extraída de Spowart, 2006).

\section{CONCLUSÕES}

$\mathrm{Na}$ aplicação em pauta, o sistema foi concebido principalmente para apresentar cenários visuais para o tomador de decisões sobre o sequenciamento de lavra, quando da ocorrência de minerais-minérios disseminados em matriz visualmente distinguível. A distribuição espacial da espécie mineralógica é gerada e seu teor é calculado para ter-se avaliação da quantidade do bem de interessa contido nestas, permitindo, portanto, uma previsão do volume total desse minério no bloco e uma visualização simulada do cenário. Pode-se, assim, comparar a simulação com o aspecto real da frente de lavra, levando a ganho na confiabilidade das inferências visuais do conteúdo de minério naquela frente, feitas pelo operador.

As funções de distribuições estatísticas para cada característica da fratura (comprimento, largura, abertura/espessura, orientação e concentração, ou seja: a distribuição espacial dentro do bloco) são fixas e cabe ao usuário apenas entrar com os parâmetros destas. Naturalmente a análise estatística desses atributos do jazimento deve ser feita anteriormente, de modo a garantir a aderência estatística dos modelos de distribuição.

O segundo exemplo de simulação aqui mostrado (uso de distribuição de Poisson, com modelo em Object Pascal), demonstra que o sistema pode se prestar a usos muito mais amplos, como aquele de caracterização de microestruturas em seções polidas, ou a análise de imagens de diversa natureza. O sistema aqui desenvolvido, portanto, pode vir a subsidiar ferramentas computacionais que podem caracterizar feições metalográficas, a exemplo daquela feita por Silva e Bonfioli (2007), a qual detecta, conta e classifica grãos isolados ou aglomerados (clusters) em imagens micrográficas.

\section{AGRADECIMENTOS}

Finalmente, os autores agradecem a suporte do Programa de Pós-graduação em Engenharia Mineral e da Pró-reitoria de Pesquisa e Pós-graduação da UFOP. O segundo autor também agradece ao CNPq pelo apoio, sob forma de bolsa de produtividade em pesquisa. 


\section{REFERÊNCIAS BIBLIOGRÁFICAS}

1. FERNANDES, C. A. B. de A. Gerenciamento de riscos em projetos: como usar o Microsoft Excel para realizar a simulação Monte Carlo. Disponível em: <http://www.bbbrothers.com.br/files/pdfs/artigos/simul_monte_carlo.pdf> (acesso em 04/05/13)

2. HAMMSERSLEY, J. M. \& HANDSCOMB, D. C. Monte Carlo Methods. 1967.

3. HASTINGS, W. K. Monte Carlo Sampling Methods Using Markov Chains and Their Applications. Biometrika 57 (1), 97, 1970.

4. HILLIER, F. S. \& LIEBERMAN, G. J. Introduction to Operations Research, 8th ed., New York: McGraw-Hill, 2005.

5. J. HROMKOVIC, Algorithms for hard problems: introduction to combinatorial optimization, randomization, approximation, and heuristics. [S.I.]: Springer-Verlag, London - Berlin Heidelberg - New York, 2001.

6. LIMA, E. L. Geometria analítica e álgebra linear, 2a ed., Rio de Janeiro: IMPA, 324 p., 2006.

7. N. METROPOLIS, A. ROSENBLUTH, M. ROSENBLUTH, A. TELLER, E. TELLER, Equation of State Calculations by Fast Computing Machines, Journal of Chemical Physics 21, 1087, 1953.

8. SILVA, A. C., BONFIOLI, L. F., Algoritmo de percolação para a deteç̧ão e contagem de clusters em imagens digitais. In: III Workshop em Computação, Universidade Federal de Ouro Preto, 2007.

9. SOBOL, I. O método de Monte Carlo. Moscou: Editorial Mir. 1983. 64 p.

10. SPOWART, J. E.; MARUYAMA, B.; MIRACLE, D. B. Multi-scale characterization of spatially heterogeneous systems: implications for discontinuously reinforced metal-matrix composite microstructures. Materials Science and Engineering A 307 (2001) 51-66.

11. SPOWART, J. E. Microstructural characterization and modeling of discontinuously-reinforced aluminum composites. Materials Science and Engineering A 425 (2006) 225-237. 